\title{
An Integrated Strategy for Cost Optimization of Reverse Logistics Network Under Uncertain Environment
}

\author{
Yunzhi Ma ${ }^{1}$, Liyun Zhang ${ }^{2}$, Xianglin $\mathrm{Lv}^{1}$, Zhengying Cai ${ }^{1, *}$ \\ ${ }^{1}$ College of Computer and Information Technology, China Three Gorges University, Yichang, China \\ ${ }^{2}$ College of Economics and Management, China Three Gorges University, Yichang, China \\ Email address: \\ 3061366583@qq.com (Yunzhi Ma),1206409954@qq.com (Liyun Zhang),1094492712@qq.com (Xianglin Lv), \\ master_cai@163.com (Zhengying Cai) \\ *Corresponding author
}

\section{To cite this article:}

Yunzhi Ma, Liyun Zhang, Xianglin Lv, Zhengying Cai. An Integrated Strategy for Cost Optimization of Reverse Logistics Network Under Uncertain Environment. International Journal of Economics, Finance and Management Sciences. Vol. 5, No. 1, 2017, pp. 24-33. doi: $10.11648 /$ j.ijefm.20170501.13

Received: October 19, 2016; Accepted: December 26, 2016; Published: December 29, 2016

\begin{abstract}
In uncertain environment, it is very difficult to optimize both cost and performance in complex reverse logistics network. This paper develops an integrated strategy to solve the cost optimization problem in reverse logistics network. First, the integrated scheme is based on the fuzzy AHP, where the cost coefficient and the demand quantities are modeled as fuzzy numbers to measure different uncertain factors. Second, the linear programming is introduced for cost optimization to calculate the operational objective function of the reverse logistics network. Third, some experiments are made to verify the proposed model. According to different uncertain factors, the optimal cost strategy can be constructed for uncertain use demand. Last, some interesting conclusions are drawn on the proposed method for decision makers to optimize the cost of the reverse logistics network, and future work direction is also provided.
\end{abstract}

Keywords: Reverse Logistics Network, Cost Optimization, Fuzzy AHP, Linear Programming

\section{Introduction}

Nowadays the resources and environment of our world are under a highly pressure, so it is importan to find better ways to reuse and utilize them. It is more challenging that how and when to send the products from the consumers to suppliers in a cost-effective manner. i.e., Jung (2016) built supply planning models for a remanufacturer under just-in-time manufacturing environment with reverse logistics [1]. In order to meet this cost challenge, an integrated strategy for cost optimization of reverse logistics network comes up with a way, providing a powerful framework, which can ensure that raw materials and finished goods could be sent in an efficient movement and timely availability. i.e., Huang (2016) introduced the condition of reverse logistics supplier selection, which is the rough set based approach to generic routing problems [2]. Cannella (2016) concerned closed-loop supply chains and the reverse logistics factors to influence performance [3].
Cost optimization problem of reverse logistics network roots in a network structure, which is consisting of a finite number of nodes and arcs attached to them. This problem is also a linear programming problem. When the cost coefficients and the supply and demand quantities are known exactly, efficient algorithms have been developed for solving the problem. Li (2016) discussed multi-objective optimization for multi-period reverse logistics network design [4]. However, sometimes these parameters may not be presented in an exact manner. For instance, in a time frame, the unit shipping cost may vary. Because of some uncontrollable factors, the supplies and demands may be uncertain. For outsourcing reverse logistics, Tavana [5] developed an integrated intuitionistic fuzzy AHP and SWOT method, the method could simultaneously satisfy the constraints and the goal to a maximal degree to derive the solution. To do quantitatively with inexact information in making decisions, Demirel (2016) evaluated a mixed integer linear programming model to optimize reverse logistics activities of end-of-life vehicles in Turkey [6], Djikanovic 
(2016) extended a new integrated forward and reverse logistics model in a case study [7], and Ayvaz (2015) gave a stochastic reverse logistics network design for the waste of electrical and electronic equipment [8].

To solve this problem, one straightforward idea is to apply the existing integer linear programming techniques $[9,10]$ to the fuzzy cost problem of reverse logistics network, since the cost problem of reverse logistics network is essentially an integer linear program. Unfortunately, a majority of the existing techniques $[11,12,13]$ only offer crisp solutions. Ferri (2015) illustrated reverse logistics network for municipal solid waste management with the inclusion of waste pickers as a Brazilian legal requirement [13]. Choudhary (2015) made a carbon market sensitive optimization model for integrated forward-reverse logistics [14], and Kilic (2015) modelled reverse logistics system design for the waste of electrical and electronic equipment (WEEE) in Turkey [15].

However, because of the structure of the cost problem in reverse logistics, the refinements of the problem parameters are required by their methods to be able to derive the bounds of the objective value in some situations. Besides, there are also researches discussing the cost optimization of reverse logistics network under uncertain environment. For a reverse logistics system with a real case application, Ozkan [16] presented a fuzzy mixed integer linear programming model and in reverse logistics through integration of GIS, AHP and integer programming. Acar [17] offered an evaluating of the location of regional return centers. For this problem, their methods are capable to determine the efficient solutions, but they can only provide crisp solutions. Silva (2015) proposed proposal for cleaner production oriented practices ecodesign and reverse logistics [18], Hsueh (2015) put forward constructing a network model to rank the optimal strategy for implementing the sorting process in reverse logistics with case study of photovoltaic industry [19], and Kim (2015) researched an integrated approach for collection network design, capacity planning and vehicle routing in reverse logistics [20].

Obviously, the total cost will be fuzzy, if the cost coefficients or the supply and demand quantities are fuzzy. Here is a solution procedure developed in this paper, which is able to compute the fuzzy objective value of the total cost of reverse logistics network under uncertain environment. In the problem, at least one of the parameters are fuzzy numbers [21, 22, 23, 24]. Under stochastic environment, Roghanian (2014) gave an optimization model for reverse logistics network by using genetic algorithm. And based on genetic algorithm [25], Liu (2014) built a network site optimization of reverse logistics for E-commerce[26]. Calculating the lower and the upper bounds of the $\theta$-level cuts of the objective value is formulating a pair of two-level mathematical programs. By enumerating different values of $\theta$, the membership function of the fuzzy objective value is derived numerically.

This paper develops an integrated strategy to solve the cost optimization problem in reverse logistics network. First, the integrated scheme is based on the fuzzy AHP, where the cost coefficient and the demand quantities are modeled as fuzzy numbers to measure different uncertain factors. Second, the integer linear programming is introduced for cost optimization to calculate the operational objective function of the reverse logistics network. Third, some experiments are made to verify the proposed model. According to different uncertain factors, the optimal cost strategy can be constructed for uncertain use demand. Last, some interesting conclusions are drawn on the proposed method for decision makers to optimize the cost of the reverse logistics network, and future work direction is also provided.

\section{Cost Optimization Problem of Reverse Logistics Network}

\subsection{An Integrated Reverse Logistics Network}

An integrated reverse logistics network can be described as shown in Figure 1. Normally, a manufacturer's product should be moved through the logistics network before it reaches the distributor or user. After the sale of the product, the logistics process is reverse, namely reverse logistics. If the product is defective, it is general that the user would select to return the product. In this case, the manufacturer would reorganize shipping of the defective product to fit the needs of user, and more work will also be involved, such as product testing, repairing, dismantling, or recycling. The reversed product apparently traveled in different direction of the supply chain network so as to recycle or reuse the defective or returned products. The logistics operation for such matters will cost time and money for both sides.

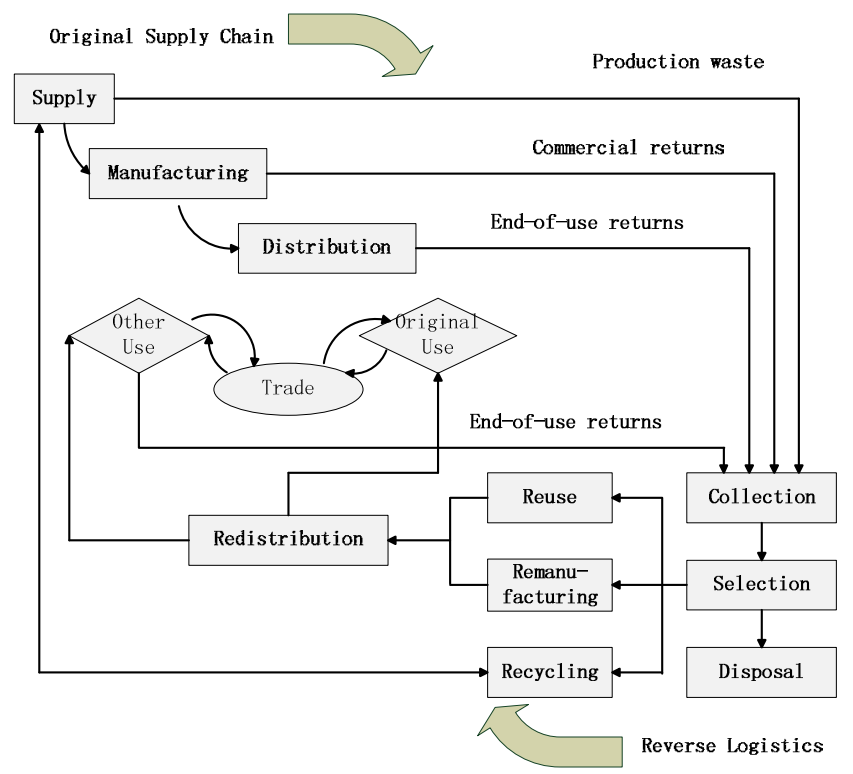

Figure 1. An integrated model for the network of reverse logistics.

Assuming a reverse logistics with $m$ suppliers and $n$ users, $h_{i}>0$ units supplied by supplier $i$ and $k_{j}>0$ units required by user $j$, there is a unit shipping cost $\mathrm{e}_{i j}$ for each 
link $(i, j)$ from supplier $i$ to user $j$. To satisfy the demand and minimize the total cost $R$, the problem is to determine a better way of shipping the available amount.

The number of units transported from supply $h_{i}$ to demand $k_{j}$ can be denoted by $w_{i j}$. At a time when the shipping costs, supplies, and demands are not known exactly, there is:

$$
\begin{aligned}
\tilde{R}=\min & \sum_{j=1}^{n} \sum_{i=1}^{m} \tilde{E}_{i j} W_{i j} \\
\text { s. t. } & \sum_{i=1}^{m} W_{i j}=\tilde{K}_{j}, \quad j=1, \ldots, n, \\
& \sum_{j=1}^{n} W_{i j}=\tilde{H}_{i}, \quad i=1, \ldots, m, \\
& W_{i j} \geq 0, \quad \forall i, j
\end{aligned}
$$

From the following pair of two-level mathematical programs, the lower are upper bounds $\tilde{R}$ at possibility level and $\theta$ can be solved easily, which is similar to the discussion of the inequality-constraint case.

$$
\begin{aligned}
& R_{\theta}^{L}=\min _{\substack{\left(E_{i j}\right)_{\theta}^{L} \leq e_{i j} \leq\left(E_{i j}\right)_{\theta}^{U} \\
\left(H_{i}\right)_{\theta}^{L} \leq h_{i} \leq\left(H_{i}\right)_{\theta}^{V} \\
\left(K_{j}\right)_{\theta}^{L} \leq k_{j} \leq\left(K_{j}\right)_{\theta}^{V} \\
\sum_{i=1}^{m} h_{i}=\sum_{j=1}^{n} k_{j} \\
\forall i, j}} \begin{cases}\min \sum_{j=1}^{n} \sum_{i=1}^{m} e_{i j} W_{i j} \\
\text { s.t. } \sum_{i=1}^{m} W_{i j}=k_{j}, \quad j=1, \ldots, n \\
\sum_{j=1}^{n} W_{i j}=h_{i}, \quad i=1, \ldots, m \\
W_{i j} \geq 0, & \forall i, j\end{cases} \\
& R_{\theta}^{U}=\max _{\substack{\left.\left(E_{i j}\right)_{\theta} \leq e_{i j} \leq\left(E_{i j}\right)_{\theta}^{U} \\
\left(H_{i}\right)_{\theta}^{L} \leq h_{j} \leq H_{i}\right)_{\theta}^{U} \\
\left(K_{j}\right)_{\theta} \leq k_{j} \leq\left(K_{j}\right)_{\theta} \\
\sum_{i=1}^{m} h_{i}=\sum_{j=1}^{n} k_{j} \\
\forall i, j}}\left\{\begin{array}{cl}
\min \sum_{j=1}^{n} \sum_{i=1}^{m} e_{i j} W_{i j} \\
\text { s.t. } \sum_{i=1}^{m} W_{i j}=k_{j}, \quad j=1, \ldots, n \\
\sum_{j=1}^{n} W_{i j}=h_{i}, \quad i=1, \ldots, m \\
W_{i j} \leq 0, \quad \forall i, j
\end{array}\right.
\end{aligned}
$$
are:

The homologous pair of one-level mathematical programs

$$
\begin{aligned}
R_{\theta}^{L}=\min & \sum_{j=1}^{n} \sum_{i=1}^{m}\left(E_{i j}\right)_{\theta}^{L} W_{i j} \\
\text { s.t. } & \sum_{i=1}^{m} W_{i j}=k_{j}, \quad j=1, \ldots, n, \\
& \sum_{j=1}^{n} W_{i j}=h_{i}, \quad i=1, \ldots, m, \\
& \sum_{j=1}^{n} k_{j}=\sum_{i=1}^{m} h_{i}, \\
& \left(K_{j}\right)_{\theta}^{L} \leq k_{j} \leq\left(K_{j}\right)_{\theta}^{U}, \quad j=1, \ldots, n, \\
& \left(H_{i}\right)_{\theta}^{L} \leq h_{i} \leq\left(H_{i}\right)_{\theta}^{U}, \quad i=1, \ldots, m, \\
W_{i j} \geq 0 & \forall i, j
\end{aligned}
$$

$$
\begin{aligned}
R_{\theta}^{U}=\max & \sum_{j=1}^{n} k_{j} t_{j}+\sum_{i=1}^{m} h_{i} s_{i} \\
\text { s. t. } & S_{i}+t_{j} \leq\left(E_{i j}\right)_{\theta}^{U} \\
& \sum_{i=1}^{m} h_{i}=\sum_{j=1}^{n} k_{j}, \\
& \left(K_{j}\right)_{\theta}^{L} \leq k_{j} \leq\left(K_{j}\right)_{\theta}^{U}, \quad j=1, \ldots, n, \\
& \left(H_{i}\right)_{\theta}^{L} \leq h_{i} \leq\left(H_{i}\right)_{\theta}^{U}, \quad i=1, \ldots, m, \\
& S_{i}, t_{j} \text { unrestricted in sign, } \forall i, j
\end{aligned}
$$

By solving Model (4) and Model (5), the lower and upper bounds of the total transportation cost at $\theta$-level can be obtained. The $\theta$-level constitutes the membership function $\varphi_{\tilde{p}}$ by sets $\left[R_{\theta}^{L}, R_{\theta}^{U}\right]$ of $\tilde{\mathrm{R}}$ at different possibility levels.

\subsection{Fuzzy Cost in Reverse Logistics}

Assuming the unit shipping cost $e_{i j}$, supply $h_{i}$, and demand $k_{j}$ can be represented as $\tilde{E}_{i j}, \tilde{H}_{i}$, and $\tilde{K}_{j}$ respectively. Note that a fuzzy set $\tilde{Q}$ is convex if $\varphi_{\tilde{Q}}\left(\lambda_{W_{1}}+(1-\lambda)_{W_{2}}\right) \geq \min \left\{\varphi_{\tilde{Q}}\left(W_{1}\right), \varphi_{\tilde{Q}}\left(W_{2}\right)\right\}, W_{1}, W_{2} \in W, \lambda \in[0,1]$. Let $\varphi_{\tilde{E}_{i j}}, \varphi_{\tilde{H}_{i}}, \varphi_{\tilde{K}_{j}}$ refer to their membership functions. There are:

$$
\begin{gathered}
\tilde{E}_{i j}=\left\{\left(e_{i j}, \varphi_{\tilde{E}_{i j}}\left(e_{i j}\right)\right) \mid e_{i j} \in S\left(\tilde{E}_{i j}\right)\right\}, \\
\tilde{H}_{i}=\left\{\left(h_{i}, \varphi_{\tilde{H}_{i}}\left(h_{i}\right)\right) \mid h_{i} \in S\left(\tilde{H}_{i}\right)\right\} \\
\tilde{K}_{j}=\left\{\left(k_{j}, \varphi_{\tilde{K}_{j}}\left(k_{j}\right)\right) \mid k_{j} \in S\left(\tilde{K}_{j}\right)\right\},
\end{gathered}
$$

The mathematical description of the common cost problem is:

$$
\begin{aligned}
R=\min & \sum_{j=1}^{n} \sum_{i=1}^{m} e_{i j} W_{i j} \\
\text { s. t. } & \sum_{i=1}^{m} W_{i j} \geq k_{j}, \quad j=1, \ldots, n \\
& \sum_{j=1}^{n} W_{i j} \leq h_{i}, \quad i=1, \ldots, m \\
& W_{i j} \geq 0, \forall i, j
\end{aligned}
$$

Intuitively, the total cost $R$ will be fuzzy, if any of the parameters $e_{i j}, h_{i}$ or $k_{j}$ is fuzzy. The conventional cost problem in reverse logistics turns into the fuzzy cost problem in reverse logistics defined in model (7).

For example, thinking of the cost problem of two suppliers $\tilde{H}_{1}=(3,5,7), h_{2}=7$ and two users $k_{1}=6$, 
$\widetilde{K}_{2}=(2,5,8)$, where $\tilde{H}_{1}$ and $\tilde{K}_{2}$ are triangular fuzzy numbers. This fuzzy cost problem can be formulated as:

$$
\begin{gathered}
\tilde{R}=\min 2 w_{11}+5 w_{12}+8 w_{21}+3 w_{22} \\
\text { s. t. } w_{11}+w_{12} \leq(3,5,7) \\
w_{21}+w_{22} \leq 7 \\
w_{12}+w_{22} \geq(2,5,8) \\
w_{11}+w_{21} \geq 6 \\
w_{11}, w_{12}, w_{21}, w_{22} \geq 0
\end{gathered}
$$

Specifically, there is,

$$
\begin{gathered}
R_{\theta=0}^{L}=\min 2 w_{11}+5 w_{12}+8 w_{21}+3 w_{22} \\
\text { s.t. } w_{11}+w_{12} \leq 3 \\
w_{21}+w_{22} \leq 7 \\
w_{12}+w_{22} \geq 2 \\
W_{11}+w_{21} \geq 6 \\
w_{11}, w_{12}, w_{21}, w_{22} \geq 0
\end{gathered}
$$

$$
\begin{gathered}
R_{\theta=0}^{U}=\max 2 w_{11}+5 w_{12}+8 w_{21}+3 w_{22} \\
\text { s.t. } w_{11}+w_{12} \leq 7 \\
w_{21}+w_{22} \leq 7 \\
W_{12}+w_{22} \geq 8 \\
W_{11}+w_{21} \geq 6 \\
W_{11}, w_{12}, w_{21}, w_{22} \geq 0
\end{gathered}
$$

Where $S\left(\tilde{E}_{i j}\right), S\left(\tilde{H}_{i}\right)$, and $S\left(\tilde{K}_{j}\right)$ are the supports of $\tilde{E}_{i j}$, $\tilde{H}_{i}$, and $\tilde{K}_{j}$, which are the universe set of the unit shipping cost, the quantity supplied by $i$ th supplier, and the quantity required by $j$ th user. The fuzzy cost problem is the following form.

$$
\begin{aligned}
\tilde{R}=\min & \sum_{j=1}^{n} \sum_{i=1}^{m} \tilde{E}_{i j} W_{i j} \\
\text { s.t. } & \sum_{i=1}^{m} W_{i j} \geq \tilde{K}_{j}, \quad j=1, \ldots, n \\
& \sum_{j=1}^{n} W_{i j} \leq \tilde{H}_{i}, \quad i=1, \ldots, m \\
& W_{i j} \geq 0, \quad \forall i, j
\end{aligned}
$$

\section{Integrated Strategy for Cost Optimization}

\subsection{Optimal Cost Strategy for Use Demand}

As the membership function of the total cost $\tilde{R}$, there is $\theta$-cuts of $\tilde{E}_{i j}, \tilde{H}_{i}$, and $\tilde{K}_{j}$ as:

$$
\begin{array}{r}
\left(E_{i j}\right)_{\theta}=\left[\left(E_{i j}\right)_{\theta}^{L},\left(E_{i j}\right)_{\theta}^{U}\right]=\left[\min _{e_{i j}}\left\{e_{i j} \in S\left(\tilde{E}_{i j}\right) \mid \varphi_{\tilde{E}_{i j}}\left(e_{i j}\right) \geq \theta\right\}, \max _{e_{i j}}\left\{e_{i j} \in S\left(\tilde{E}_{i j}\right) \mid \varphi_{\tilde{E}_{i j}}\left(e_{i j}\right) \geq \theta\right\}\right] \\
\left(H_{i}\right)_{\theta}=\left[\left(H_{i}\right)_{\theta}^{L},\left(H_{i}\right)_{\theta}^{U}\right]=\left[\min _{s_{i}}\left\{h_{i} \in S\left(\tilde{H}_{i}\right) \mid \varphi_{\tilde{H}_{i}}\left(h_{i}\right) \geq \theta\right\}, \max _{h_{i}}\left\{h_{i} \in S\left(\tilde{H}_{i}\right) \mid \varphi_{\tilde{H}_{i}}\left(h_{i}\right) \geq \theta\right\}\right] \\
\left(K_{j}\right)_{\theta}=\left[\left(K_{j}\right)_{\theta}^{L},\left(K_{j}\right)_{\theta}^{U}\right]=\left[\min _{k_{j}}\left\{k_{j} \in S\left(\tilde{K}_{j}\right) \mid \varphi_{\tilde{K}_{j}}\left(k_{j}\right) \geq \theta\right\}, \max _{k_{j}}\left\{k_{j} \in S\left(\tilde{K}_{j}\right) \mid \varphi_{\tilde{K}_{j}}\left(k_{j}\right) \geq \theta\right\}\right]
\end{array}
$$

On account of the fuzzy AHP, the membership function $\varphi_{\tilde{R}}$ is:

$$
\boldsymbol{\varphi}_{\tilde{R}}(r)=\sup _{e, h, k} \min \left\{\varphi_{\tilde{E}_{i j}}\left(e_{i j}\right), \boldsymbol{\varphi}_{\tilde{H}_{i}}\left(h_{i}\right), \varphi_{\tilde{K}_{j}}\left(k_{j}\right), \forall i, j \mid r=R(\boldsymbol{e}, h, k)\right\}
$$

where $R(\boldsymbol{e}, h, k)$ is defined in model (7).

In Eq. (12), it involves several membership functions. It is hardly possible to derive $\varphi_{\tilde{p}}$ in closed form. According to (12), the minimum of $\varphi_{\tilde{E}_{i j}}, \varphi_{\tilde{H}_{i}}$, and $\varphi_{\tilde{K}_{j}}, \forall i, j$ is $\varphi_{\tilde{R}}$. In order to satisfy $\varphi_{\tilde{R}}(r)=\theta, \varphi_{\tilde{E}_{i j}}\left(e_{i j}\right) \geq \theta, \varphi_{\tilde{H}_{i}}\left(h_{i}\right) \geq \theta$, $\varphi_{\tilde{K}_{j}}\left(k_{j}\right) \geq \theta$ and at least one $\varphi_{\tilde{E}_{i j}}\left(e_{i j}\right), \varphi_{\tilde{H}_{i}}\left(h_{i}\right)$, or
$\varphi_{\tilde{K}_{j}}\left(k_{j}\right), \forall i, j$, equal to $\theta$ such that $r=R(\boldsymbol{e}, h, k)$. To find the membership function $\varphi_{\bar{R}}$, it is necessary to find the right shape function of $\varphi_{\bar{R}}$, with lower bound $R_{\theta}^{L}$ and upper bound $R_{\theta}^{U}$ of the $\theta$-cut of $\tilde{R}$. The minimum and the maximum of $R(\boldsymbol{e}, h, k)$ is $R_{\theta}^{L}$ and $R_{\theta}^{U}$, respectively. 


$$
\begin{gathered}
R_{\theta}^{L}=\min \left\{R(\boldsymbol{e}, h, k) \mid\left(E_{i j}\right)_{\theta}^{L} \leq e_{i j} \leq\left(E_{i j}\right)_{\theta}^{U},\left(H_{i}\right)_{\theta}^{L} \leq h_{i} \leq\left(H_{i}\right)_{\theta}^{U},\left(K_{j}\right)_{\theta}^{L} \leq k_{j} \leq\left(K_{j}\right)_{\theta}^{U}, \forall i, j\right\} \\
R_{\theta}^{U}=\max \left\{R(\boldsymbol{e}, h, k) \mid\left(E_{i j}\right)_{\theta}^{L} \leq e_{i j} \leq\left(E_{i j}\right)_{\theta}^{U},\left(H_{i}\right)_{\theta}^{L} \leq h_{i} \leq\left(H_{i}\right)_{\theta}^{U},\left(K_{j}\right)_{\theta}^{L} \leq k_{j} \leq\left(K_{j}\right)_{\theta}^{U}, \forall i, j\right\}
\end{gathered}
$$

\subsection{Solving Step for Integrated Strategy}

For model (13) and model (14), there are $\sum_{j=1}^{n} k_{j} \geq \sum_{i=1}^{m} h_{i}$ in the range of $\left[\left(K_{i}\right)_{\theta}^{L},\left(K_{i}\right)_{\theta}^{U}\right]$, and $\left[\left(H_{j}\right)_{\theta}^{L},\left(H_{j}\right)_{\theta}^{U}\right]$ respectively.

However, it is necessary that $\sum_{i=1}^{m} k_{i} \geq \sum_{j=1}^{n} h_{j}$ in reverse logistics network. Hence, model (13) and model (14) become:

$$
\begin{aligned}
& R_{\theta}^{L}=\min _{\left(E_{i j}\right)_{\theta}^{L} \leq e_{i j} \leq\left(E_{i j}\right)_{\theta}^{U}}\left\{\sum_{j=1}^{n} \sum_{i=1}^{m} e_{i j} w_{i j}\right\} \\
& \left(H_{i}\right)_{\theta}^{L_{0} \leq h_{i} \leq\left(H_{i}\right)_{\theta}^{U}} \\
& \left(K_{j}\right)_{\theta}^{U} \leq k_{j} \leq\left(K_{j}\right)_{\theta}^{U} \\
& \sum_{i=1}^{m} h_{i} \geq \sum_{j i, j}^{n} k_{j} \\
& \text { s.t. } \sum_{i=1}^{m} w_{i j} \leq k_{j}, \quad j=1, \ldots, n \\
& \sum_{j=1}^{n} w_{i j} \geq h_{i}, \quad i=1, \ldots, m \\
& w_{i j} \geq 0, \quad \forall i, j \\
& R_{\theta}^{U}=\max _{\left(E_{i j}\right)_{\theta}^{L} \leq e \leq\left(E_{i j}\right)_{\theta}^{U}}\left\{\sum_{j=1}^{n} \sum_{i=1}^{m} e_{i j} w_{i j}\right\} \\
& \left(H_{i}\right)_{\theta}^{U} \leq h_{i} \leq\left(H_{i}\right)_{\theta}^{U} \\
& \left(K_{j}\right)_{\theta}^{L} \leq k_{j} \leq\left(K_{j}\right)_{\theta}^{U} \\
& \sum_{i=1}^{m} h_{i} \geq \sum_{j i, j}^{n} k_{j} \\
& \text { s.t. } \sum_{i=1}^{m} w_{i j} \leq k_{j}, j=1, \ldots, n \\
& \sum_{j=1}^{n} w_{i j} \leq h_{i}, i=1, \ldots, m \\
& w_{i j} \leq 0, \quad \forall i, j
\end{aligned}
$$

If $\sum_{j=1}^{n}\left(K_{j}\right)_{\theta=0}^{L} \leq \sum_{i=1}^{m}\left(H_{i}\right)_{\theta=0}^{U}$, model (15) will be infeasible for any $\theta$-level.

The problem is formulated to become a maximization problem to be consistent with the maximization operation of level 1 to solve model (16), which is well-known from the duality theorem of integer linear programming that the primal model and the dual model have the same objective value. Then, model (16) becomes:

$$
\begin{gathered}
R_{q}^{U}=\max _{\substack{\left(E_{i j}\right)_{q}^{L} \leqslant e_{i j} \leqslant\left(E_{i j}\right)_{q}^{U} \\
\left(H_{i}\right)_{q}^{L} \leqslant h_{i} \leqslant\left(H_{i}\right)_{q}^{U} \\
\left(K_{j}\right)_{q}^{q} \leqslant k_{j} \leqslant\left(K_{j}\right)_{q}^{U} \\
\sum_{i=1}^{m} h_{i} \geqslant \sum_{j=1}^{n} k_{j} \\
\forall i, j}}\left\{\sum_{j=1}^{m} h_{i} s_{i}+\sum_{j=1}^{n} k_{j} t_{j}\right\} \\
\text { s.t. }-s_{i}+t_{j} \leqslant e_{i j}, i=1, \ldots, m, j=1, \ldots, n \\
s_{i}, t_{j} \geqslant 0 n \quad \forall i, j
\end{gathered}
$$

Since $\left(\mathrm{E}_{i j}\right)_{\theta}^{L} \leq e_{i j} \leq\left(E_{i j}\right)_{\theta}^{U}, \forall i, j$, by setting $e_{i j}$ to its upper bound $\left(E_{i j}\right)_{\theta}^{U}, \forall i, j$ in model (17), one can derive the upper bound of the objective value, because this gives the largest feasible region.

Then, the model (17) is changed as :

$$
\begin{aligned}
& R_{\theta}^{U}=\max _{\substack{\left(H_{i}\right)_{\theta^{\prime}} \leq h_{i} \leq\left(H_{i}\right)_{\theta}^{U} \\
\left(K_{j}\right)_{\theta} \leq k_{j} \leq\left(K_{j}\right)_{\theta}^{U}}}\left\{\sum_{i=1}^{m} h_{i} s_{i}+\sum_{j=1}^{n} k_{j} t_{j}\right\} \\
& \quad \sum_{i=1}^{m} h_{i} \geq \sum_{j i j}^{n} k_{j} \\
& \text { s.t. }- S_{i}+t_{j} \leq\left(E_{i j}\right)_{\theta}^{U}, i=1, \ldots, m, j=1, \ldots, n \\
& s_{i}, t_{j} \geq 0, \quad \forall i, j
\end{aligned}
$$

Hence, model (18) can be reformulated as also:

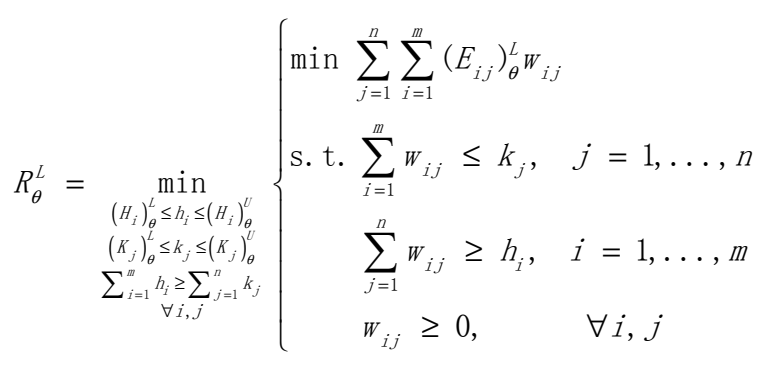

Hence, there are:

$$
\begin{aligned}
R_{\theta}^{L}=\min & \sum_{j=1}^{n} \sum_{i=1}^{m}\left(E_{i j}\right)_{\theta}^{L} W_{i j} \\
\text { s.t. } & \sum_{j=1}^{n} w_{i j} \geq k_{j}, \quad j=1, \ldots, n, \\
& \sum_{i=1}^{m} w_{i j} \leq h_{i}, \quad i=1, \ldots, m, \\
& \sum_{i=1}^{m} h_{i} \geq \sum_{j=1}^{n} k_{j}, \\
& \left(K_{j}\right)_{\theta}^{L} \leq k_{j} \leq\left(K_{j}\right)_{\theta}^{U}, \quad j=1, \ldots, n, \\
& \left(H_{i}\right)_{\theta}^{L} \leq h_{i} \leq\left(H_{i}\right)_{\theta}^{U}, \quad i=1, \ldots, m, \\
& W_{i j} \geq 0, \quad \forall i, j
\end{aligned}
$$




$$
\begin{aligned}
R_{\theta}^{U}=\max & -\sum_{i=1}^{m} h_{i} S_{i}+\sum_{j=1}^{n} k_{j} t_{j} \\
\text { s.t. } & -S_{i}+t_{j} \leq\left(E_{i j}\right)_{\theta}^{U}, \quad i=1, \ldots, m, \quad j=1, \ldots, n \\
& \sum_{i=1}^{m} h_{i} \geq \sum_{j=1}^{n} k_{j} \\
& \left(K_{j}\right)_{\theta}^{L} \leq k_{j} \leq\left(K_{j}\right)_{\theta}^{U}, \quad j=1, \ldots, n, \\
& \left(H_{i}\right)_{\theta}^{L} \leq h_{i} \leq\left(H_{i}\right)_{\theta}^{U}, \quad i=1, \ldots, m \\
& S_{i}, t_{j} \geq 0, \quad \forall i, j
\end{aligned}
$$

If $\sum_{i=1}^{m}\left(H_{i}\right)_{\theta=0}^{U} \leq \sum_{j=1}^{n}\left(K_{j}\right)_{\theta=0}^{L}$, problems (4) and (5) are assured to be feasible. The problem will be infeasible only if this condition is not satisfied. Just like the conventional cost problem can be assumed to make the problem feasible, a fictitious supply point $m+1$ with an account of $h_{m+1} \geq \sum_{i=1}^{m}\left(H_{i}\right)_{\theta=0}^{U}-\sum_{j=1}^{n}\left(K_{j}\right)_{\theta=0}^{L}$ is also feasible in this case.

The feasible regions defined by $\theta_{1}$ in models (20) and (21) are smaller than those defined by $\theta_{2}$ for two possibility levels $\theta_{1}$ and $\theta_{2}$, so $0<\theta_{1}<\theta_{2} \leq 1$. Consequently, $(R)_{\theta_{1}}^{L} \geq(R)_{\theta_{2}}^{L}$ and $(R)_{\theta_{1}}^{U} \leq(R)_{\theta_{2}}^{U}$.

This property, which is based on the definition of convex rough set, assures the convexity of $\tilde{R}$. This provides us a feasible solution for the optimization of revers logistics in uncertain environment. According to $L(r)$ and $R(r)$, the function $\varphi_{\tilde{R}}$ is changed as:

$$
\boldsymbol{\varphi}_{\tilde{R}}= \begin{cases}L(r), & (R)_{\theta=0}^{L} \leq r \leq(R)_{\theta=1}^{L} \\ 1, & (R)_{\theta=1}^{L} \leq r \leq(R)_{\theta=1}^{U} \\ R(r), & (R)_{\theta=1}^{U} \leq r \leq(R)_{\theta=0}^{U}\end{cases}
$$

At different possibility levels of $\theta$, the numerical solutions for $R_{\theta}^{L}$ and $R_{\theta}^{U}$ can be collected to approximate the functions of $L(r)$ and $R(r)$.

\section{Experimental Analysis}

\subsection{Problem Description}

To verify the proposed model, a numerical example is presented here, which is derived from the logistics case in reality. For simplification of analysis, the cost problem can be considered with three fuzzy demands, two fuzzy supplies and one fuzzy shipping cost to explain the proposed approach. Demand 3 and Supply 1 are triangular fuzzy numbers. In order to assure the feasibility of $\sum_{i=1}^{m} h_{i}=\sum_{j=1}^{k} k_{j}$, the total demand must be equal to or less than the total supply.
The main initial parameters are as follows. For the $\theta=0$ cut of $\tilde{R}$, the lower bound of $R^{*}=410$ appears at $W_{11}^{*}=0.4, w_{12}^{*}=w_{13}^{*}=0.3, w_{21}^{*}=w_{22}^{*}=w_{23}^{*}=0.3$ with $k_{1}=40, k_{2}=30, k_{3}=50$ and $h_{1}=80$, $h_{2}=50$, while the upper bound of $R^{*}=800$ appears at $W_{11}^{*}=w_{12}^{*}=0.4, w_{13}^{*}=0.2, w_{21}^{*}=w_{22}^{*}=0.2$, $w_{23}^{*}=0.5$, with $k_{1}=40, k_{2}=40, k_{3}=90$ and $h_{1}=110, h_{2}=50$.

Another utmost end of $\theta=1$, the lower bound of $R^{*}=520$ arises at $w_{11}^{*}=0.5, w_{12}^{*}=w_{13}^{*}=0.25$, $W_{21}^{*}=W_{22}^{*}=W_{23}^{*}=0.3$ with $k_{1}=50, k_{2}=40$, $k_{3}=60$ and $h_{1}=80, h_{2}=60$, as well as, the upper bound of $R^{*}=600$ arises at $W_{11}^{*}=0.6, W_{12}^{*}=0.3$, $W_{13}^{*}=0.1, w_{21}^{*}=W_{22}^{*}=0.3, w_{23}^{*}=0.4$ with $k_{1}=60, k_{2}=50, k_{3}=60$ and $h_{1}=100, h_{2}=60$.

So this problem can be described by the following form:

$$
\begin{gathered}
\tilde{R}=\min 20 w_{11}+60 w_{12}+90 w_{13}+70 w_{21}+70 w_{22}+30 w_{23} \\
\text { s.t. } W_{21}+w_{22}+w_{23} \leq(50,70,80,90) \\
w_{11}+w_{12}+w_{13} \leq(80,100,110) \\
W_{13}+w_{23} \geq(50,60,90) \\
W_{12}+w_{22} \geq(30,40,50,60) \\
W_{11}+w_{21} \geq(40,50,60,80) \\
W_{11}, W_{12}, w_{13}, w_{21}, w_{22}, w_{23} \geq 0
\end{gathered}
$$

Figure 2 shows that the total demand is $\widetilde{K}_{1}+\widetilde{K}_{2}+\widetilde{K}_{3}=(120,150,170,230)$ and the total supply is $\tilde{H}_{1}+\tilde{H}_{2}=(130,170,180,210)$.

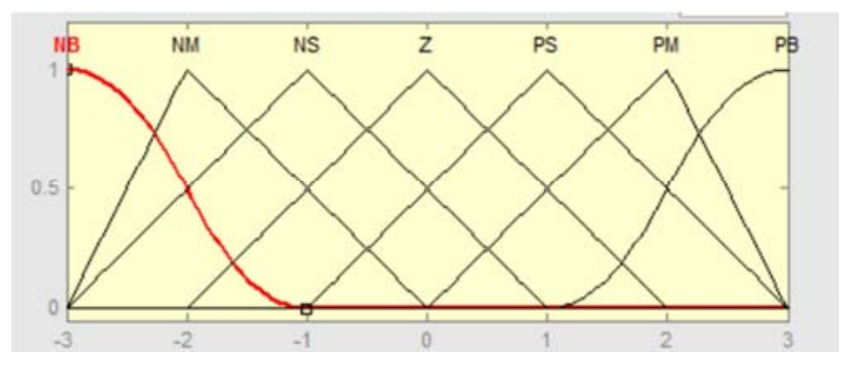

Figure 2. The membership functions of $\tilde{R}$.

That implys the problem is feasible, with the lower and upper bounds of $\tilde{R}$ is: 


$$
\begin{aligned}
& R_{\theta}^{L}=\min \left\{20 W_{11}+60 W_{12}+90 W_{13}+(70+10 \theta)_{W_{21}}+70 W_{22}+30 W_{23}\right\} \\
& \text { s. t. } w_{21}+w_{22}+w_{23} \leq h_{2} \\
& w_{11}+w_{12}+w_{13} \leq h_{1} \\
& W_{13}+w_{23} \geq k_{3} \\
& W_{12}+W_{22} \geq k_{2} \\
& w_{11}+w_{21} \geq k_{1} \\
& h_{1}+h_{2} \geq k_{1}+k_{2}+k_{3} \\
& 40+10 \theta \leq k_{1} \leq 80-20 \theta, \\
& 30+10 \theta \leq k_{2} \leq 60-10 \theta, \\
& 50+10 \theta \leq k_{3} \leq 90-30 \theta, \\
& 80+20 \theta \leq h_{1} \leq 110-10 \theta, \\
& 50+20 \theta \leq h_{2} \leq 90-10 \theta, \\
& W_{11}, W_{12}, w_{13}, W_{21}, W_{22}, W_{23} \geq 0 \\
& R_{\theta}^{U}=\max \left\{h_{1} S_{1}+h_{2} S_{2}+k_{1} t_{1}+k_{2} t_{2}+k_{3} t_{3}\right\} \\
& \text { s.t. } s_{1}+t_{3} \leq 90 \\
& s_{1}+t_{2} \leq 60 \\
& s_{1}+t_{1} \leq 20 \\
& s_{2}+t_{3} \leq 30 \\
& s_{2}+t_{2} \leq 70 \\
& s_{2}+t_{1} \leq(100-10 \theta) \\
& h_{1}+h_{2} \geq k_{1}+k_{2}+k_{3} \\
& 40+10 \theta \leq k_{1} \leq 80-20 \theta \text {, } \\
& 30+10 \theta \leq k_{2} \leq 60-10 \theta \text {, } \\
& 50+10 \theta \leq k_{3} \leq 90-30 \theta \text {, } \\
& 80+20 \theta \leq h_{1} \leq 110-10 \theta, \\
& 50+20 \theta \leq h_{2} \leq 90-10 \theta, \\
& s_{1}, s_{2}, t_{1}, t_{2}, t_{3} \geq 0
\end{aligned}
$$

The logistics performance and cost of the lower and upper bounds of $\tilde{R}$ possibility level $\theta$ are shown in Figure 3 and Figure 4.

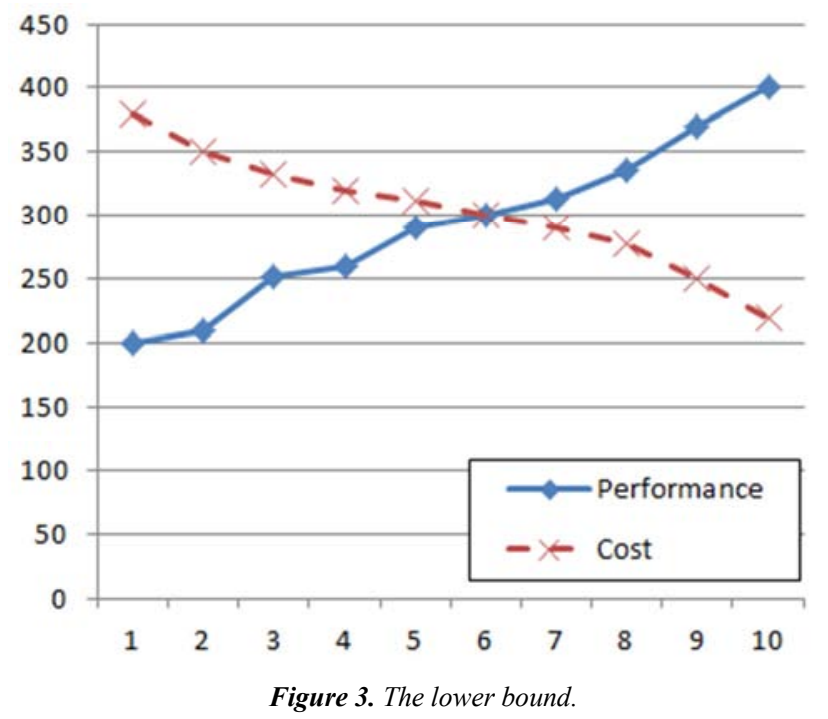

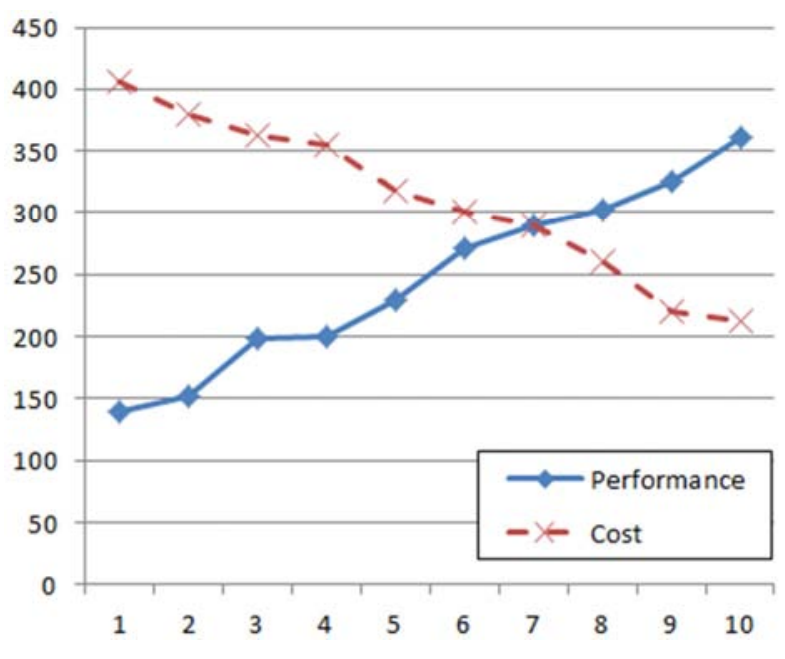

Figure 4. The upper bound.

From Figure 3 and Figure 4, when the cost is decreased, the performance of reverse logistics is totally increased. The different values of $\theta$ have been cataloged as $[0,1.0]$. The probability of the transportation cost will emerge in the associated range in uncertain environment and be represented by the $\theta$-cut of $\tilde{R}$.

Especially, the $\theta=1.0$ cut displays the most likely of the total cost. At the same time, the cut $\theta=0$ reveals the possible range of the total cost. At the fuzzy cost in this example, it's possible that the cost falls at the range of 380 and 410 but is impossible to fall outside of 220 and 210 .

The membership function $\varphi_{\tilde{R}}$ of this example has been curve labeled at Figure 2 of fuzzy membership functions.

$$
\begin{aligned}
R= & \min \sum_{j=1}^{n} \sum_{i=1}^{m} e_{i j} w_{i j} \\
\text { s.t. } & \sum_{i=1}^{m} w_{i j}=k_{j}, j=1, \ldots, n, \\
& \sum_{j=1}^{n} w_{i j}=h_{i}, i=1, \ldots, m, \\
& w_{i j} \geq 0, \forall i, j
\end{aligned}
$$

It is worthy taking note that the maximum total shipping quantity interrelated to the total cost do not need to be the highest. In this example, 180 is the largest probable measurement to be shipped and also is the largest total supply, it means the optimization of the reverse logistics network.

\subsection{Result Analysis}

For further analysis, it is assumed that the equality constraints take the place of inequality constraints, there is 


$$
\begin{gathered}
\tilde{R}=\min 20 w_{11}+60 w_{12}+90 w_{13}+80 w_{21}+70 w_{22}+30 w_{23} \\
\text { s.t. } w_{21}+w_{22}+w_{23}=(50,70,80,90) \\
w_{11}+w_{12}+w_{13}=(80,100,110) \\
w_{12}+w_{22}=(30,40,50,60) \\
W_{11}+w_{21}=(40,50,60,90) \\
W_{13}+w_{23}=(50,60,90) \\
W_{11}, W_{12}, w_{13}, w_{21}, w_{22}, w_{23} \geq 0
\end{gathered}
$$

The following pair of mathematical programs figures out the lower and upper bounds of the $\theta$-cut of $\tilde{R}$ :

$$
\begin{aligned}
& R_{\theta}^{L}=\min 20 W_{11}+60 W_{12}+90 W_{13}+(70+10 \theta)_{W_{21}}+70 W_{22}+30 W_{23} \\
& \text { s. t. } W_{21}+w_{22}+w_{23}=h_{2} \\
& W_{11}+W_{12}+w_{13}=h_{1} \\
& W_{13}+w_{23}=k_{3} \\
& W_{12}+W_{22}=k_{2} \\
& W_{11}+w_{21}=k_{1} \\
& h_{1}+h_{2}=k_{1}+k_{2}+k_{3} \\
& 40+10 \theta \leq k_{1} \leq 80-20 \theta \text {, } \\
& 30+10 \theta \leq k_{2} \leq 60-10 \theta \text {, } \\
& 50+10 \theta \leq k_{3} \leq 90-30 \theta \text {, } \\
& 80+20 \theta \leq h_{1} \leq 110-10 \theta, \\
& 50+20 \theta \leq h_{2} \leq 90-10 \theta \text {, } \\
& W_{11}, W_{12}, W_{13}, W_{21}, W_{22}, W_{23} \geq 0 \\
& R_{\theta}^{U}=\max h_{1} S_{1}+h_{2} S_{2}+k_{1} t_{1}+k_{2} t_{2}+k_{3} t_{3} \\
& \text { s. t. } s_{1}+t_{2} \leq 60 \\
& s_{1}+t_{1} \leq 20 \\
& s_{1}+t_{3} \leq 90 \\
& s_{2}+t_{1} \leq 30 \\
& S_{2}+t_{3} \leq(100-10 \theta) \\
& s_{2}+t_{2} \leq 70 \\
& h_{1}+h_{2}=k_{1}+k_{2}+k_{3} \\
& 40+10 \theta \leq k_{1} \leq 80-20 \theta \text {, } \\
& 30+10 \theta \leq k_{2} \leq 60-10 \theta \text {, } \\
& 50+10 \theta \leq k_{3} \leq 90-30 \theta, \\
& 80+20 \theta \leq h_{1} \leq 110-10 \theta \text {, } \\
& 50+20 \theta \leq h_{2} \leq 90-10 \theta \text {, } \\
& s_{1}, s_{2}, t_{1}, t_{2}, t_{3} \text { unrestricted in sign }
\end{aligned}
$$

The bounds of the total cost at eleven $\theta$-cuts are listed in Figure 5 and Figure 6.

From Figure 5 and Figure 6, when the cost is decreased, the performance of reverse logistics is increased. It also has a membership degree of 0.93 , corresponding to the crossing of the right shape function of the total demand and the left shape function of the total supply.

$\varphi_{\tilde{R}}$, the membership function in this example is the curve labeled as Equality-constraints in Figure 2. The problem is infeasible at the time when $\theta$ is greater than 0 , which means, when the maximum degree is equal to 0.9 , the constraints could be satisfied. Because of equality constraints are more restrictive, the objective value's membership function of this example is contained.

Note that this point. At $\theta=0$, the lower bound of the objective value is 200, which appears at $w_{11}^{*}=0.6$, $w_{12}^{*}=0.3, w_{13}^{*}=0.1, w_{21}^{*}=0.3, w_{22}^{*}=0.2$, $W_{23}^{*}=0.5$, with $k_{1}=60, k_{2}=30, k_{3}=50$ and $h_{1}=80, h_{2}=50$.
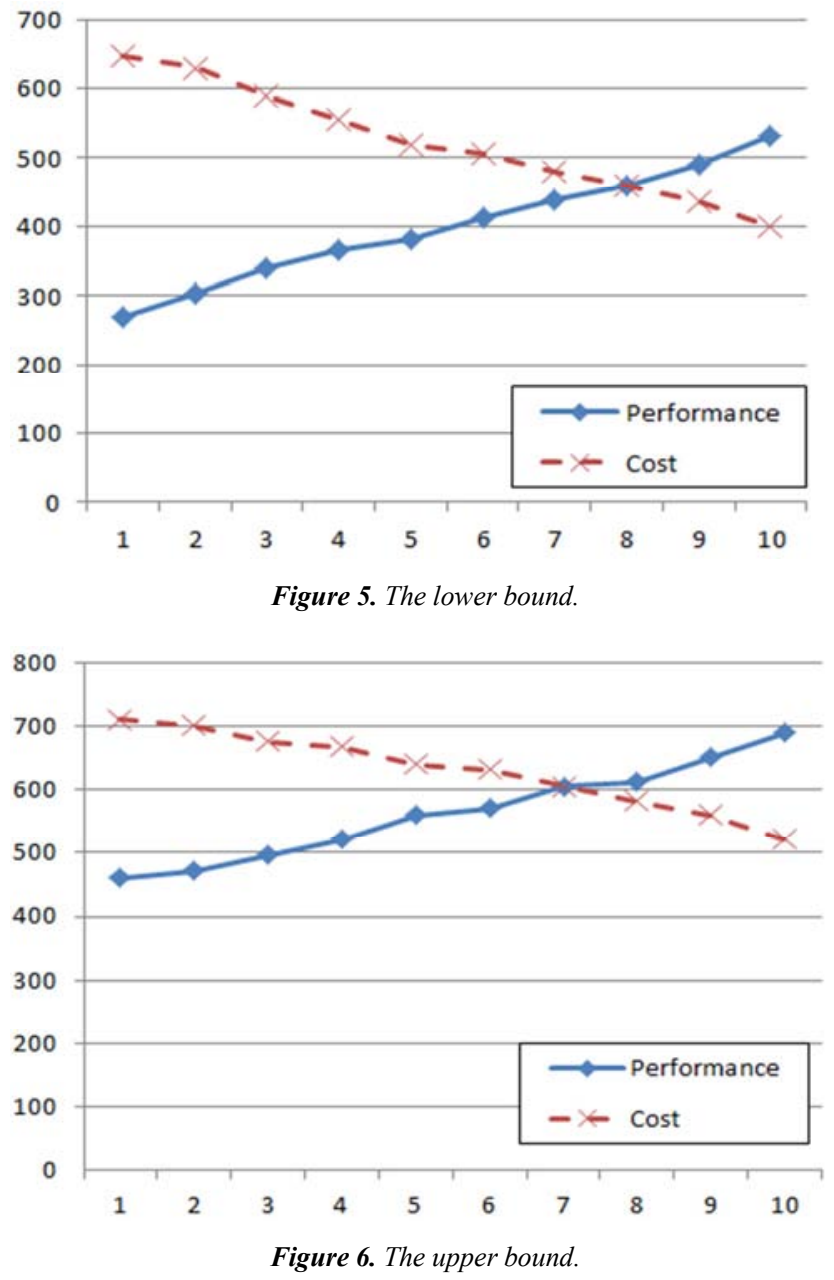

Meanwhile, the upper bound is 800, appearing at $w_{11}^{*}=0.3, w_{12}^{*}=0.3, w_{13}^{*}=0.4, w_{21}^{*}=0.4$, $w_{22}^{*}=0.4, w_{23}^{*}=0.2$ with $k_{1}=40, k_{2}=40$, $k_{3}=90$ and $h_{1}=110, h_{2}=50.740$ is the single point of $\theta$-cut at $\theta=0.9$. The optimized solution is $w_{11}^{*}=0.3$, $W_{12}^{*}=0.3, W_{13}^{*}=0.4$.

The membership function is derived numerically and mathematical form is not provided in this study. The inequality constraints and equality constraints of the fuzzy cost problem are compared in Figure 7. 


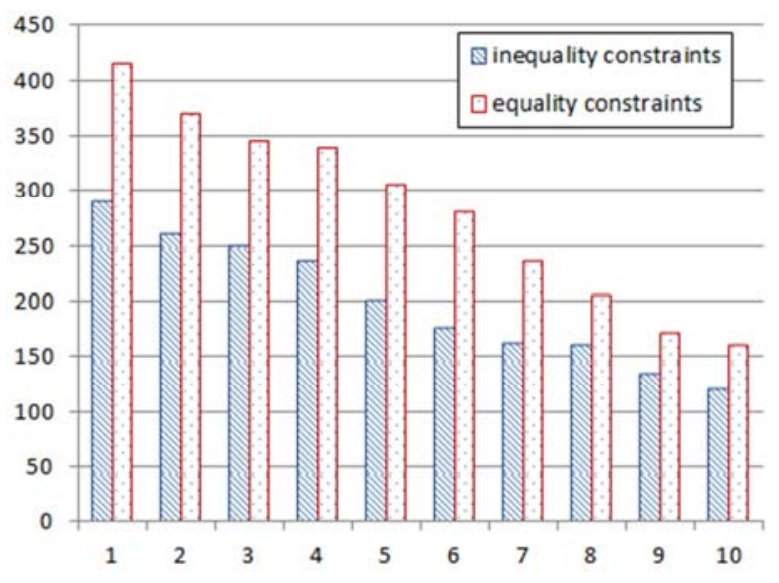

Figure 7. Inequality constraints and equality constraints of the fuzzy cost problem.

It is apparent that both inequality constraints and equality constraints can optimize the cost problem in reverse logistics network by fuzzy measurement, though taking more advantage in equity constraints. Therefore, the $\theta$-cut is the only way to approximate the membership degree of a specific transportation cost.

It's challenging to derive the mathematical form of the membership function for directly calculating the membership degree. The membership function of the objective value of the inequality problem includes that of the equality problem, in that the equality constraints are more restrained than inequality constraints. In practice, the structure of the cost problem is quite complex. Because of that, the highest total transportation cost may not appear when the total quantity transported is the largest.

\section{Conclusion}

Different from those studies derived the objective values in crisp values, an integrated strategy for cost optimization of reverse logistics network under uncertain environment is modeled here with fuzzy total cost. When the demand quantities, the supply quantities, and the unit shipping costs are fuzzy numbers, it provides us a helpful tool to optimize both cost and performance in complex reverse logistics network in uncertain environment. The fuzzy AHP is used to reform the fuzzy cost problem for practicablel solution. The fuzzy objective value's lower and upper bounds of the $\theta$-cuts can be calculated by enumerating different $\theta$ values to rough the membership function. Sometimes the crisp values of obtained results may not lead to some helpful information.

Furthermore, future work would focus on considering more uncontrollable factors, the system parameters of reverse logistics network in real world applications which may not be known exactly. And more information should be provided for decision making by using different membership functions to express the practical parameters.

\section{Acknowledgment}

This research was supported by the National Natural
Science Foundation of China (No. 71471102), and Science and Technology Research Program, Hubei Provincial Department of Education in China (Grant No. D20101203).

\section{References}

[1] Jung, KS; Dawande, M; Geismar, HN; Guide, VDR; Sriskandarajah, C, Supply planning models for a remanufacturer under just-in-time manufacturing environment with reverse logistics, Annals of Operations Research, 240 (2016), 533-581.

[2] Huang, CC; Liang, WY; Tseng, TL; Chen, PH, The rough set based approach to generic routing problems: case of reverse logistics supplier selection, Journal of Intelligent Manufacturing, 27 (2016), 781-795.

[3] Cannella, S; Bruccoleri, M; Framinan, JM, Closed-loop supply chains: What reverse logistics factors influence performance? International Journal of Production Economics, 175 (2016), 35-49.

[4] Li, S; Wang, NM; Jia, T; He, ZW; Liang, HG, Multi-objective Optimization for Multi-period Reverse Logistics Network Design, IEEE Transactions on Engineering Management, 63 (2016), 223-236.

[5] Tavana, M; Zareinejad, M; Di Caprio, D; Kaviani, MA, An integrated intuitionistic fuzzy AHP and SWOT method for outsourcing reverse logistics, Applied Soft Computing, 40 (2016), 544-557.

[6] Demirel, E; Demirel, N; Gokcen, H, A mixed integer linear programming model to optimize reverse logistics activities of end-of-life vehicles in Turkey, Journal of Cleaner Productioyuchi, 112 (2016), 2101-2113.

[7] Djikanovic, J; Vujosevic, M, A new integrated forward and reverse logistics model: A case study, International Journal of Computational Intelligence Systems, 9 (2016), 25-35.

[8] Ayvaz, B; Bolat, B; Aydin, N, Stochastic reverse logistics network design for waste of electrical and electronic equipment, Resources Conservation and Recycling, 104 (2015), 391-404.

[9] Lee, JE; Chung, KY; Lee, KD; Gen, M, A multi-objective hybrid genetic algorithm to minimize the total cost and delivery tardiness in a reverse logistics, Multimedia Tools and Applications, 74 (2015), 9067-9085.

[10] Moghaddam, KS, Fuzzy multi-objective model for supplier selection and order allocation in reverse logistics systems under supply and demand uncertainty, Expert Systems with Applications, 42 (2015), 6237-6254.

[11] Yanik, S, Reverse logistics network design under the rsk of hazardous materials transportation, Human and Ecological Risk Assessment, 21 (2015), 1277-1298.

[12] Zhou, XG; Zhou, YH, Designing a multi-echelon reverse logistics operation and network: A case study of office paper in Beijing, Resources Conservation and Recycling, 100 (2015), 58-69.

[13] Ferri, GL; Chaves, GDD; Ribeiro, GM, Reverse logistics network for municipal solid waste management: The inclusion of waste pickers as a Brazilian legal requirement, Waste Management, 40 (2015), 173-191. 
[14] Choudhary, A; Sarkar, S; Settur, S; Tiwari, MK, A carbon market sensitive optimization model for integrated forwardreverse logistics, International Journal of Production Economics, 164 (2015), 433-444.

[15] Kilic, HS; Cebeci, U; Ayhan, MB, Reverse logistics system design for the waste of electrical and electronic equipment (WEEE) in Turkey, Resources Conservation and Recycling, 95 (2015), 120-132.

[16] Ozkan, B; Basligil, H; Kaya, I; Ozkir, V, A fuzzy mixed integer linear programming model for a reverse logistics system with a real case application, Journal of Multiple-valued Logic and Soft Computing, 25 (2 3) 2015, 269-289.

[17] Acar, AZ; Onden, I; Kara, K, Evaluating the location of regional return centers in reverse logistics through integration of gis, ahp and integer programming, International Journal of Industrial Engineering-theory Applications and Practice, 22 (4) $2015,399-411$.

[18] Silva, ALE; Moraes, JAR; Machado, EL, Proposal for cleaner production oriented practices ecodesign and reverse logistics, Engenharia Sanitaria E Ambiental, 20 (1) 2015, 29-46.

[19] Hsueh, JT; Lin, CY, Constructing a network model to rank the optimal strategy for implementing the sorting process in reverse logistics: case study of photovoltaic industry, Clean Technologies and Environmental Policy, 17 (1) 2015, 155-174.

[20] Kim, JS; Lee, DH, An integrated approach for collection network design, capacity planning and vehicle routing in reverse logistics, Journal of The Operational Research Society, 66 (1) 2015, 76-85.
[21] Alumur, SA; Tari, I, Collection center location with equity considerations in reverse logistics network, Infor, 52 (4) 2014, 157-173.

[22] Eskandarpour, M; Masehian, E; Soltani, R; Khosrojerdi, A, A reverse logistics network for recovery systems and a robust metaheuristic solution approach, International Journal of Advanced Manufacturing Technology, 74 (9-12) 2014, 13931406.

[23] Ramos, TRP; Gomes, MI; Barbosa, Povoa, AP, Planning a sustainable reverse logistics system: Balancing costs with environmental and social concerns, Omega-international Journal of Management Science, 48 (2014), 60-74.

[24] Niknejad, A; Petrovic, D, Optimization of integrated reverse logistics networks with different product recovery routes, European Journal of Operational Research, 238 (1) 2014, 143154.

[25] Roghanian, E; Pazhoheshfar, P, An optimization model for reverse logistics network under stochastic environment by using genetic algorithm, Journal of Manufacturing Systems, 33 (3) 2014, 348-356.

[26] Liu, DW, Network site optimization of reverse logistics for Ecommerce based on genetic algorithm, Neural Computing \& Applications, 25 (1) 2014, 67-71.

[27] Subulan, K; Baykasoglu, A; Saltabas, A, An improved decoding procedure and seeker optimization algorithm for reverse logistics network design problem, Journal of Intelligent \& Fuzzy Systems, 27 (6) 2014, 2703-2714. 